\title{
Ion Channels and Bone Homeostasis Imbalance
}

\author{
Dian Jiao, Shuai Guo, Yafei Chen, Yong Zhan and Hailong An* \\ Key Laboratory of Molecular Biophysics, Hebei Province, Institute of Biophysics, School of Sciences, China
}

*Corresponding author: Hailong An, Key Laboratory of Molecular Biophysics, Hebei Province, Institute of Biophysics, School of Sciences, China

\begin{tabular}{l}
\hline ARTICLE INFO \\
\hline Received: 业 March 20, 2019 \\
Published: 业 March 28, 2019 \\
\hline Citation: Dian Jiao, Shuai Guo, Yafei \\
Chen, Yong Zhan, Hailong An. Ion Chan- \\
nels and Bone Homeostasis Imbalance. \\
Biomed J Sci \& Tech Res 16(3)-2019. \\
BJSTR. MS.ID.002862.
\end{tabular}

Keywords: Bone homeostasis; Ion channel; Osteoblast; Osteoclast
ABSTRACT

To sustain the bone weight, there is a delicate balance between the osteoblast bone formation and osteoclast bone resorption known as bone homeostasis. The imbalance of bone homeostasis will induce bone disorder, such as osteoporosis and osteopetrosis. Numerous studies have shown that ion channels play critical roles in maintaining the bone homeostasis. Dysfunction of ion channels accompanied with disorder of the osteoblast bone formation and osteoclast bone resorption. In the present paper, the authors discussed the physiological roles of bone homeostasis on human body and summarized the ion channels expressing on the membrane of osteoblasts and osteoclasts. Finally, the limitations of the prior art and the prospects in this field were discussed.

Abbreviations: ENaC: Epithelial Sodium Channels; ALP: Alkaline Phosphatase; cGMP: Cyclic guanosine monophosphate; CLC: Chloride Channels; CLIC: Chloride Intracellular Channel; CFTR: Cystic Fibrosis Transmembrane Conductance Regulator; KCC: Potassium Chloride Cotransporter

\section{Introduction}

The human bone environment undergoes remodeling through bone formation and bone resorption throughout life to maintain bone homeostasis [1], which means bone resorption of osteoclasts is always accompanied with bone formation of osteoblasts [2]. The tight balance between bone formation and bone resorption regulates bone development and bone homeostasis. When the balance between bone formation and bone resorption is disrupted, it may lead to pathological conditions such as osteoporosis and osteopetrosis, whose characteristic may respectively be low bone mass and high bone mass, resulting in increased bone fragility and increased risk of fracture. As transmembrane proteins on cell membrane, ion channels are the basic excitatory units on the tissue cell membrane and participate in the process of transmitting electrical signals, which can be regarded as an excitable protein molecule reacts to specific stimuli. Depending on the permeability of the ions, the ion channels can be classified according to specific ions.

\section{Sodium Ion Channels}

Sodium ions widely exist in extracellular fluids and are the most abundant cations in human body, which are most closely related to liquid osmotic pressure. The voltage-gated sodium ion channel is a highly glycosylated complex consisting of an alpha subunit and two beta subunits. When the membrane potential is maintained at the resting membrane potential, the channel is closed, when the membrane depolarization potential is above the threshold, channel opened. The voltage-gated sodium ion channel is opened by depolarization, which allows sodium ions to flow in, causing membrane depolarization and inducing action potential [3]. It has been reported that there are two sodium channels in osteoblasts, namely voltage-sensitive sodium channels (Nav) and epithelial sodium channels (ENaC). In 1994, Killick R first discovered the ENaC channel expresses on cartilage and osteoblasts, plays a critical role in perceptual acidosis, maintenance of sodium homeostasis and transduction of mechanical stimulation. As a novel non-voltagedependent sodium channel, ENaC is a member of the ENaC/ degenerin family and transports sodium ions to critical pathways in epithelial cells, vascular endothelial cells and other tissues, which can be inhibited by amiloride. At the molecular level, ENaC consists of three homologous subunits, $\alpha, \beta$, and $\gamma$ [4]. Wherein each subunit consists of two transmembrane helices and one extracellular loop, and has a similar secondary structure, through a large extracellular 
domain, which linked to the two membrane spanning domains (TM1 and TM2), and short intracellular $\mathrm{N}$ - and $\mathrm{C}$ - terminal composition $[5,6]$. The crystal structure and site-directed mutagenesis of ENaC indicate that $\mathrm{ENaC}$ has a central ion channel located on the central axis of symmetry of the three subunits [7].

In many excitable cells, the $\mathrm{Na}+$ channel is responsible for the depolarization phase of the action potential. It is now known to identify rapidly activated $\mathrm{Na}^{+}$conductance in chicken osteoclasts [8]. This inward current is rapidly activated at membrane potential, more positive than $-30 \mathrm{mV}$ (peak amplitude is $1-2 \mathrm{~ms}$ ) and then rapidly inactivated (within $5-7 \mathrm{~ms}$ ), the kinetics of $\mathrm{ENaC}$ is faster than other voltage-gated channels in osteoclasts. The $\mathrm{Na}^{+}$current can be inhibited by nanomolar concentrations of tetrodotoxin, a blocker of certain voltage-gated $\mathrm{Na}^{+}$channels. In chicken osteoclasts, voltage-activated $\mathrm{Na}^{+}$conductance at the whole cell level is relatively small compared to conductance of other channels. This low density channel and its transient activation do not allow the generation of action potentials. In other cells, $\mathrm{Na}^{+}$conductance plays a vital role in secretion [9] and may play a role in the regulation of cell proliferation [10]. It is conceivable that larger $\mathrm{Na}^{+}$ conductance plays a role in the proliferation of pre-osteoclasts and that only residues remaining in differentiated chicken osteoclasts. The researchers also found that the $\mathrm{ENaC}-\alpha$ were expressed in rat osteoblast-like cell line (UMR-106) and primary human osteoblasts [11] and after the ENaC gene was silenced on rat osteoblasts by siRNA technique, they found that osteogenic gene-related genes' expressions decreased (Alp, Col1); after the osteogenic estradiol added to the primary cultured rat osteoblasts and mouse osteoblasts, the expression of ENaC mRNA was increased, which proved that $\mathrm{ENaC}$ in osteoblasts participated in the bone formation [4]. Some scholars have also studied the effects of ferulic acid acting on rat skull proliferation and differentiation by CCK-8 and alkaline phosphatase (ALP) staining, Cyclic guanosine monophosphate (cGMP)-dependent protein kinase II (PKGII) expression was silenced by small interfering RNA (siRNA) and then mRNA expression was detected by semi-quantitative PCR. The researchers found that ferulic acid promotes proliferation, differentiation and mineralization in rat calvarial osteoblasts in vitro, via cGMP-PKGIIENaC signaling pathway, which also enhanced expressions of osteogenic genes [12]. JL G et al. [13] also confirmed in 2017 that epithelial sodium channels can promote osteogenic differentiation through the cGmp/PKGII/ENaC signaling pathway.

In 2016, $\mathrm{Hu}$ Songyan found that an inhibitor of ENaC, amiloride, which reduced the number of TRAP-positive osteoclasts and inhibited the formation of osteoclasts by staining with tartrateresistant acid phosphatase (TRAP) and bone resorption, founding the expression of the osteoclast-specific gene CK reduced, thereby demonstrating the expression on $\mathrm{ENaC}$ osteoclasts at the cellular level and regulating osteoclast differentiation and bone resorption, further indicating that $\mathrm{ENaC}$ may be involved in osteoclast function adjust [14]. Sandra J et al. [15] using immunocytochemistry and
RNA sequencing found that voltage-gated sodium channel $\mathrm{NaV}$ channel was expressed in primary osteoblasts infant mouse skull. The carbamazepine and phenytoin, which are the $\mathrm{NaV}$ channel blockers, sensitive sodium currents were recorded by whole cell patch clamp recording.

\section{Chloride Ion Channels}

As the most important anions in human body, chloride ion plays an important role in the electrochemical balance between the intracellular and extracellular side. It also participates in regulating intracellular and extracellular functions through the active transport of chloride channel, which can regulate the physiological process through the change of its channel current, such as liquid secretion, cell volume regulation, transmembrane transport, excitatory conduction and intracellular acidification. It is reported that a variety of chloride channels exist in the cell membrane or organelles. Abnormal chloride channels can cause a variety of physiological diseases, such as cystic fibrosis, congenital myotonia, epilepsy, Barter syndrome, etc [16]. Chloride channels associated with bone metabolism have been reported to include chloride channels (CLC), chloride intracellular channel (CLIC), cystic fibrosis transmembrane conductance regulator (CFTR) and potassiumchloride cotransporter (KCC). Among above, CLC is a gated chloride channel. The chloride channel members in the CLC family usually play important roles in regulating electrical excitability and transepithelial cell transport. The CLC chloride channels exist both in the plasma membrane and the membrane of organelles.

As a member of the CLC family, ClC-3 chloride channel has been reported expressed in bone cells, and it may not only participate in cell proliferation and apoptosis of osteoclasts, but also promote new bone growth by osteoblasts [17]. At the same time, ClC- 3 chloride channel expressed in mouse osteoblast lineage cells and plays an important role in improving the mineralization ability of osteoblasts in vitro and promoting osteoblast differentiation [18]. By overexpressing $\mathrm{ClC}-3$, the expression of osteogenic markers (Alp, Ibsp and Bglap) was also elevated, which was also contributed to the calcification ability of MC3T3-E1 cells. Knockdown ClC-3 may reversed, demonstrating ClC-3 exists in osteoblasts and contributes to osteogenic differentiation [19]. Wang $\mathrm{H}$ et al. proposed the expression of Clcn3, Clcn4 and Clcn5 in MC3T3-E1 cells. Wang H et al. also suggested that in the process of bone differentiation, static compression of MC3T3-E1 cells increased the expression of ClC3 and further promoted the expression of osteogenic genes. This proves that the chloride channel $\mathrm{ClC}-3$ is involved in the mechanical stimulation of MC3T3-E1 cells [20].

Furthermore, ClC-7, located in the lysosome, is critical for the function of osteoclasts, which secretes chloride ions through the wrinkle edge of osteoclasts to secrete hydrogen ions through $\mathrm{V}-\mathrm{H}^{+}-$ ATPases to dissolve bone minerals in bone. Highly expressed in the sputum, ClC-7 can be inserted into the edges of the wrinkles. Lysosomal function changes in mice lacking ClC-7, which leads to 
severe lysosomal storage [21]. Mice lacking Clcn7 gene presents severe osteopetrosis due to the inability of osteoclasts to secrete acid, retinal degeneration and death within 7 weeks. Clcn mutation was identified in human, and homozygous mutations resulted in osteosclerosis in malignant infants [22]. Compared with other osteosclerosis mouse models, lacking ClC-7 does not affect the generation of osteoclasts attached to bone surface. $\mathrm{Clcn}^{-/}$mouse osteoclasts exhibit functional and morphological abnormalities that do not degrade bone, which is related to their ability to acidify bone and osteoclasts and then seal extracellular space. In osteoblasts, the expression of $\mathrm{ClC}-7$ is expressed by wrinkles formed by fusion of vesicles containing $\mathrm{H}^{+}$-ATPase, and protons are secreted into the voids. Clcn7 is expressed by wrinkles formed by fusion of vesicles containing $\mathrm{H}^{+}$-ATPase, and then protons are secreted into the voids in osteoblast. Mutations in Clcn7 have been identified in patients with malignant osteopetrosis in human infants [23]. In 2010, Stefanie Weinert et al. converted ClC-7, which produces mouse with point mutations, into an unconjugated (unc) $\mathrm{Cl}$ - conductor, despite maintaining lysosomal conductance and normal lysosomal $\mathrm{pH}$.

Clcn $7 \%$ mice show lysosomal storage diseases, however, their osteosclerosis is mild and they lack the coat color phenotype, only certain effects of $\mathrm{ClC}-7$ mediated $\mathrm{Cl}^{-} / \mathrm{H}^{+}$exchange and it can be taken over by $\mathrm{Cl}$ - conductance. The data show that $\mathrm{ClC}-7$ may mediate $\mathrm{Cl}^{-} / \mathrm{H}^{+}$exchange during lysosomal acidification [24]. Located in the apical membrane of epithelial cells, CFTR is a hub for transport, fluid flow and ion concentration across epithelial salts. Low bone mass and increased risk of fracture are complications of cystic fibrosis (CF), which is characterized by undecoupled bone turnover - osteoblastic bone formation damage and enhanced osteoclastic bone resorption. Intestinal malabsorption, vitamin D deficiency and inflammatory cytokines contribute to CFBD. Cftr gene knockout mice exhibited abnormal bone development and metabolism, such as reduced bone length and reduced cancellous bone volume [25]. And Le Heron L et al. [26] found that common bone deficiency disease in patients with cystic fibrosis, Cftr mRNA and protein were expressed in primary human osteoblasts.

\section{ATP-Gated Channel}

ATP is a key energy currency and a ubiquitous extracellular messenger, and it also plays an important role in bone tissue. Based on its dose and the participating purinergic receptor (P2R) subtypes, ATP can trigger many different cellular responses, including cell proliferation, differentiation, and apoptosis [27]. In the biological activities of bone tissue, ATP can be involved in bone growth, development and repair [28]. Depending on the mode of receptor transduction, P2 receptors can be divided into two families, P2X and P2Y. Among them, P2X is a family of ion receptors, which is a ligand-gated ion channel, while P2Y is a family of metabotropic receptors, belonging to G protein-coupled receptors. $\mathrm{P} 2 \mathrm{X}$ receptor is also expressed in human and mediates a variety of functions, including muscle contraction, neuronal excitation and bone formation, with subunits of intracellular N- and C- termini, two transmembrane domains and an extracellular ligand bind to the loop, but has no crystal structure. The effect of P2X on osteoblast is mainly to regulate the release of cytokines. P2X also regulates the start of osteoblast apoptosis on osteoclast [29]. It is reported that seven different $\mathrm{P} 2$ receptor subtypes were expressed in osteoblasts, including P2X2, P2X5, P2X7, P2Y1, P2Y2, P2Y4 and P2Y6. In the same time, Gallagher and Buckley reported that P2Y2 was strongly expressed in human osteoblast, while P2Y1 receptors were more predominant in rat osteoblast [30].

Shuxing et al. [31] analyzed the fitting effect of P2 receptor family in MC3T3-E1 cells by fluorescence quenching and Hill equation. Among them, the authors used a combination of Hill equations for each receptor to fit experimental data revealing that P2Y1 and P2Y7 mediate the increase in [Ca2+]i at very low and high ATP concentrations, respectively. It not only analyzes the expression of $\mathrm{P} 2$ receptor in osteoblasts, but also the interaction of individual P2 receptors in the overall response to extracellular ATP. Relevant data indicate that P2X3 and P2X2/receptor antagonist A-317491 (5-[(3-phenoxyphenyl) methyl-[(1S)-1,2,3,4tetrahydronaphthalene-1-Carbamoyl] benzene-1,2,4-tricarboxylic acid] transiently attenuates bone cancer-induced pain in mice but has no effect in the later stages of the malignant process [32]. It has also been found that the $\mathrm{P} 2 \mathrm{X} 3$ receptor is upregulated in the dorsal root ganglion function in a rat model of bone cancer [33]. It has also been reported that cancer-related bone pain is associated with osteoclast activation and $\mathrm{Ca}^{2+}$ imbalance $[34,35]$.

\section{Transient Receptor Potential Channel, TRP}

It is well known that both the external $\mathrm{Ca}^{2+}$ and intracellular $\mathrm{Ca}^{2+}$ signaling are critical to bone homeostasis.

First, the normal function of bone depends on normal serum calcium levels, while bone also plays an important role in maintaining systemic calcium homeostasis. In fact, 99\% of calcium in body is stored in bone, which contributes to its mechanical structural properties. Therefore, bone needs enough calcium to maintain bone integrity. What's more, intracellular $\mathrm{Ca}^{2+}$ is also an important second messenger in bone. Intracellular $\mathrm{Ca}^{2+}$ signaling in osteoblasts, osteoclasts, chondrocytes, and nerve endings has been shown to regulate many functions, including differentiation, signal transduction and mechanical transport, permeation and perception of painful stimuli. Therefore, fine-tuning of intracellular $\mathrm{Ca}^{2+}$ levels is critical for normal bone homeostasis, at the same time, transporters abnormalities, which are also involved in $\mathrm{Ca}^{2+}$ signaling, will clearly lead to diseases that also affect bone structure or function [36]. Maintenance of extracellular and intracellular $\mathrm{Ca}^{2+}$ homeostasis is important for bone biology and is largely dependent on $\mathrm{Ca}^{2+}$ channels. There are several types of $\mathrm{Ca}^{2+}$ channels, including

a) The ryanodine receptor (RyR) and the inositol-1,4,5triphosphate receptor (IP3R), mediates the release of $\mathrm{Ca}^{2+}$ from the endoplasmic reticulum (ER). 
b) Storage-operated calcium channels (SOCE), including ORAI1 and STIM1, mediates flux of extracellular $\mathrm{Ca}^{2+}$ into ER via ORAI1 when STIM1 detects depletion of intracellular stores,

c) Voltage-gated $\mathrm{Ca}^{2+}$ channels (VGCC) ), allows $\mathrm{Ca}^{2+}$ to flow into cells for depolarization

d) A stretch-activated $\mathrm{Ca}^{2+}$ channel mediates $\mathrm{Ca}^{2+}$ influx after mechanical stimulation

e) A transient receptor potential (TRP) family for cations [37].

It has been reported that TRPV6 is necessary to ensure adequate intestinal calcium absorption during dietary calcium deprivation and thus prevent excessive reduction of bone mineralization [38]. These findings also highlight the importance of proper dietary calcium absorption in bone. The bone abnormalities during normal calcium intake indicate that TRPV6 indirectly affects bone only by participating in intestinal calcium transport. However, TRPV6 expressed in osteoblasts and osteoclasts, although the expression levels are very low $[39,40]$, it theoretically possible directly regulated their differentiation or function. However, recent studies have convincingly demonstrated that TRPV6 does not participate in osteogenic $\mathrm{Ca}^{2+}$ uptake or mineralization [41]. Together, these results confirm TRPV6 lack of a direct role in bone metabolism. In conclusion, TRPV6 affects bone metabolism by promoting intestinal calcium transport and is particularly desirable during dietary calcium deficiency. On the other hand, in osteoclasts, in vitro studies have shown that TRP channels may be one of the Ca2+ entry pathways leading to $\mathrm{Ca}^{2+}$ oscillation [42]. It is also found that the cortical bone mass of TRPV5-/- mice reduced, which was associated with an increase in the number of osteoclasts, although bone resorption parameters are reduced [43]. These results convincingly demonstrate that TRPV5 is important for systemic calcium homeostasis by fine-tuning renal calcium reabsorption. Skeletal defects in TRPV5-/- mice may be due to abnormal external calcium balance or change in osteoclast $\mathrm{Ca}^{2+}$ signaling. It is worth noting that TRPV5 didn't express in osteoblasts. Moreover, in osteoclasts, TRPV5 is located at the edge of the wrinkles and may contribute to bone resorption. According to van der Eerden, in vitro differentiation of hematopoietic precursors isolated from TRPV5 null mice resulted in more and larger osteoclasts with severely impaired resorption capacity. TRPV5 and TRPV6 are known highly selective channels for calcium ions [44].

Rossi showed that TRPV1 expressed in osteoclasts in vitro and in vivo, which demonstrated that TRPV1 antagonist I-RTX inhibits human osteoclast formation in vitro [45], while TRPV1 agonist resin toxin increases osteoclast formation. Thereafter, Aymen I. Idris examined the effects of TRPV1 ion channel antagonist capsazepine on mouse osteoclast and osteoblast differentiation and osteoporosis induced by ovariectomy in vivo. They found that adding capsazepine, a pharmacological blockade of ion channels, also is a TRPV1 blocker. TRPV1 may have an inhibitory effect on osteoclastic bone resorption which can protect mice from oophorectomy-induced bone loss, meanwhile may also inhibit osteoblast activity and bone formation [46]. TRPV4 is a preferred cation channel for $\mathrm{Ca}^{2+}$, originally characterized by the conversion of osmotic pressure. TRPV4-mediates $\mathrm{Ca}^{2+}$ signaling in response to cartilage osmotic fluctuations, which is a potential mechanism for chondrocytes. Recent studies have shown that TRPV4 signaling plays a crucial role in skeletal development, and the loss of gene coding for mouse TRPV4 leads to accelerated aging of joint degradation [47]. Christopher J O'Conor detected the effect of TRPV4 deficiency on the intrinsic ability of bone marrow stem cells (MSCs) and adipose-derived stem cells to detect the phenotype of Trpv $4 \%$ mice and the function of TRPV4 at cellular level. Linkages further confirm that the lack of TRPV4-mediated signaling in the presence of obesity catabolism, biomechanics and inflammatory factors accelerates the progression of osteoarthritis in OA's highfat diet model [48]. Transient receptor potential vanilloid channel 2 (TRPV2) was significantly expressed in RANKL-treated RAW264.7 cells (pre-osteoclasts). Calcium oscillation in pre-osteoclast RAW264.7 cells was activated by RANKL-dependent TRPV2 and activated intracellularly calcium oscillation at the same time, and then activation of osteogenesis by NFATc1 [49].

\section{Prospective}

Ion channel is one of the basic ways to exchange and transmit signals between cells, which have become the drug targets for research in biology, medicine, pharmacy, etc. A large number of research reports indicate that ion channels can also be used as research for the treatment of osteoporosis. Many scholars have demonstrated that the function of differentiation and proliferation of osteoblasts osteoclasts can be regulated by ion channels.

However, there are several problems and phenomena:

a) The ion channels existing in bone tissue are not unique and may be affected by other channels while studying.

b) In molecular biology experiments, due to in vitro cell experiments, it has been separated from the original environment and its internal environment has also changed accordingly. It has changed from the in vivo situation, and the in vitro experiment cannot accurately explain the research mechanism. But with the development of technology and thinking, these problems will eventually be solved.

\section{Acknowledgement}

National Natural Science Foundation of China, Grant/Award Numbers: 11735006, 31400711, 31600594; Natural Science Foundation of Hebei Province of China, Grant/Award Number: C2018202302; Youth Talent Support Program of Hebei Province of China, Grant/Award Number: 2013001. 


\section{References}

1. Seeman E, Delmas PD (2006) Bone quality-the material and structural basis of bone strength and fragility. N Engl J Med 354: 2250-2261.

2. Henriksen K, Bollerslev J, Everts V, Karsdal MA (2011) Osteoclast activity and subtypes as function of physiology and pathology-implications for future treatments of osteoporosis. Endocr Rev 32: 31-63.

3. Chen Wei, Lu Li, Li Qingnan (2010) Progress in research on sodium channel of epithelial cells in osteoblasts[J]. Chinese Journal of Osteoporosis 16(6).

4. Loffing J, Schild L (2005) Functional domains of the epithelial sodium channel. J Am Soc Nephrol 16(11): 3175-3181.

5. Kashlan OB, Kleyman TR (2011) ENaC structure and function in the wake of a resolved structure of a family member. Am J Physiol Renal Physiol 301(4): F684-696.

6. Bhalla V, Hallows KR (2008) Mechanisms of ENaC regulation and clinical implications. J Am Soc Nephrol 19(10):1845-1854.

7. Edelheit O, Ben Shahar R, Dascal N, Hanukoglu A, Hanukoglu I (2014) Conserved charged residues at the surface and interface of epithelia sodium channel subunits - Roles in cell surface expression and the sodium self-inhibition response. FEBS J 281(8): 2097-2111.

8. Gáspár R, AF W, Krasznai Z (1995) Tetrodotoxin-sensitive fast Na current in embryonic chicken osteoclasts[J]. Pflugers Archiv 430(4): 596-598.

9. Stein WD (1990) Channels, Carriers, and Pumps. In Stein WD(Eds.), (2 $2^{\text {nd }}$ edn.). Academic Press: San Diego, USA.

10. Rooj AK, McNicholas CM, Bartoszewski R, et al. (2012) Glioma-specific Cation Conductance Regulates Migration and Cell Cycle Progression. The journal of biological chemistry 287(6): 4053-4065.

11. Kizer N, Guo XL, Hruska K (1997) Reconstitution of stretch-activated cation channels by expression of the $\alpha$-subunit of the epithelial sodium channel cloned from osteoblasts. Proc Natl Acad Sci 94(3): 1013-1018.

12. Chen J, Zhang H, Zhang X (2014) Epithelial sodium channel enhanced osteogenesis via cGMP/PKGII/ENaC signaling in rat osteoblast. Mol Biol Rep 41(4): 2161-2169.

13. JL G, Chen J, GZ Y (2017) Ferulic acid induces proliferation and differentiation of rat osteoblasts in vitro through cGMP/PKGII/ENaC signaling. J Asian Nat Prod Res 19(2): 176-187.

14. Hu Songyan, Jin Xiaodong, Zhang Hao, et al. (2016) Effects of epithelia sodium channel on rat osteoclast differentiation and bone resorption function. Journal of Southern Medical University 2016(8).

15. Petty SJ, Milligan CJ, Todaro M (2016) The antiepileptic medications carbamazepine and phenytoin inhibit native sodium currents in murine osteoblasts. Epilepsia 57(9): 1398-1405.

16. Patil V, Gupta S (2016) Studies on Chloride Channels and their Modulators. Current Topics in Medicinal Chemistry 16(16): 1862-1876.

17. Okamoto F, Kajiya H, Toh K (2008) Intracellular ClC-3 chloride channels promote bone resorption in vitro through organelle acidification in mouse osteoclasts. Am J Physiol Cell Physiol 294(3): C693-701.

18. QC L, DJ N, LJ R (2015) Chloride-hydrogen antiporters ClC-3 and ClC 5 drive osteoblast mineralization and regulate fine-structure bone patterning in vitro. Physiological Reports 3(11): e12607.

19. Lu X, Ding Y, Niu Q (2017) ClC-3 chloride channel mediates the role of parathyroid hormone on osteogenic differentiation of osteoblasts. PLoS ONE 12(4): e0176196.

20. Wang H, Huo N, Li F (2010) Osteogenic role of endosomal chloride channels in MC3T3-E1 cells. Mol Cell Biochem 342(1-2): 191-199.

21. Kasper D, Cases RP, Fuhrmann JC (2005) Loss of the chloride channel ClC-7 leads to lysosomal storage disease and neurodegeneration. The EMBO Journal 24(5): 1079-1091.
22. Kornak U, Kasper D, Bosl MR (2001) Loss of the ClC-7 chloride channel leads to osteopetrosis in mice and man. Cell 104(2): 205-215.

23. Deng H, He D, Rong P (2016) Novel CLCN7 mutation identified in a Han Chinese family with autosomal dominant osteopetrosis-2. Mol Pain 12: 174480 .

24. Weinert S, Jabs S, Supanchart C (2010) Lysosomal Pathology and Osteopetrosis upon Loss of $\mathrm{H}+$-Driven Lysosomal Cl- Accumulation. Science 2010(328).

25. Yamamoto Mizuma S, Wang GX, Hume JR (2004) P2Y purinergic receptor regulation of CFTR chloride channels in mouse cardiac myocytes. J Physiol 556(Pt 3): 727-737.

26. Le Heron L, Guillaume C, Velard F (2010) Cystic fibrosis transmembrane conductance regulator (CFTR) regulates the production of osteoprotegerin (OPG) and prostaglandin (PG) E(2) in human bone. J Cyst Fibros 9(1): 69-72.

27. IR 0, ML K, Brandao Burch A (2012) The regulation of osteoblast function and bone mineralisation by extracellular nucleotides: The role of p2x receptors. Bone 51(3): 389-400.

28. Cabahug Zuckerman P, RF S, RJ M (2018) Potential role for a specialized $\beta$ integrin-based structure on osteocyte processes in bone mechanosensation. J Orthop Res 36(2): 642-652.

29. JA G, KA B (2002) Expression and function of P2 receptors in bone. J Musculoskelet Neuronal Interact 2(5): 432-439.

30. Xing S, MW G, PH G (2016) Modeling Interactions among Individual P2 Receptors to Explain Complex Response Patterns over a Wide Range of ATP Concentrations. Front Physiol 7: 294.

31. Hansen RR, Nasser A, Falk S, Baldvinsson SB, Ohlsson PH, et al. (2012) Chronic administration of the selective $\mathrm{P} 2 \mathrm{X} 3, \mathrm{P} 2 \mathrm{X} 2 / 3$ receptor antagonist, A-317491, transiently attenuates cancer-induced bone pain in mice. Eur J Pharmacol 688(1-3): 27-34.

32. Wu JX, Xu MY, Miao XR, Lu ZJ, Yuan XM, et al. (2012) Functional upregulation of $\mathrm{P} 2 \mathrm{X} 3$ receptors in dorsal root ganglion in a rat model of bone cancer pain. Eur J Pain 16(10): 1378-1388.

33. Liu M, Yang H, Fang D, Yang JJ, Cai J, et al. (2013) Upregulation of P2X3 receptors by neuronal calcium sensor protein VILIP-1 in dorsal root ganglions contributes to the bone cancer pain in rats. Pain 154(9): 15511568.

34. Luger NM, Mach DB, Sevcik MA, Mantyh PW (2005) Bone cancer pain: from model to mechanism to therapy. J Pain Symptom Manage 29 (Suppl): S32-S46.

35. Nagae M, Hiraga T, Wakabayashi H, Wang L, Iwata K, et al. (2006) Osteoclasts play a part in pain due to the inflammation adjacent to bone. Bone 39(5): 1107-1115.

36. Blair HC, Schlesinger PH, Huang CL, Zaidi M (2007) Calcium signalling and calcium transport in bone disease. Subcell Biochem 45: 539-562.

37. Robinson LJ, Mancarella S, Songsawad D, Tourkova IL, Barnett JB, et al. (2012) Gene disruption of the calcium channel Orai1 results in inhibition of osteoclast and osteoblast differentiation and impairs skeletal development. Lab Invest 92(7): 1071-1083.

38. Lieben L, BS B, Ajibade D (2010) Trpv6 mediates intestinal calcium absorption during calcium restriction and contributes to bone homeostasis. Bone 47(2): 301-308

39. Lieben L, Carmeliet G (2012) The Involvement of TRP Channels in Bone Homeostasis[J]. Front Endocrinol (Lausanne) 3: 99.

40. Little R, Muimo R, Robson L, Harris K, Grabowski PS (2011) The transient receptor potential ion channel TRPV6 is expressed at low levels in osteoblasts and has little role in osteoblast calcium uptake. PLoS ONE 6(11): e28166.

41. Van der Eerden BC, Weissgerber P, Fratzl Zelman N, Olausson J, Hoenderop JG, et al. (2012) The transient receptor potential channel 
TRPV6 is dynamically expressed in bone cells but is not crucial for bone mineralization in mice. J Cell Physiol 227(5): 1951-1959.

42. Hwang SY, Putney JW (2011) Orai1-mediated calcium entry plays a critical role in osteoclast differentiation and function by regulating activation of the transcription factor NFATc1. FASEB J 26(4): 1484-1492

43. Hoenderop JG, Van Leeuwen JP, Van Der Eerden BC, Kersten FF, Van Der Kemp AW, et al. (2003) Renal $\mathrm{Ca}^{2+}$ wasting, hyperabsorption, and reduced bone thickness in mice lacking TRPV5. J Clin Invest 112(12): 1906-1914.

44. Van Der Eerden BC, Hoenderop JG, De Vries TJ, Schoenmaker T, Buur man CJ, et al. (2005) The epithelial Ca2+ channel TRPV5 is essential for proper osteoclastic bone resorption. Proc Natl Acad Sci USA 102(48): 17507-17512.

45. Rossi F, Bellini G, Torella M (2014) The genetic ablation or pharmacological inhibition of TRPV1 signalling is beneficial for the

\section{ISSN: 2574-1241}

DOI: 10.26717/BJSTR.2019.16.002862

Hailong An. Biomed J Sci \& Tech Res

(C) This work is licensed under Creative

Commons Attribution 4.0 License

Submission Link: https://biomedres.us/submit-manuscript.php restoration of quiescent osteoclast activity in ovariectomized mice. Br J Pharmacol 171(10): 2621-2630.

46. Idris AI, Landao Bassonga E, Ralston SH (2010) The TRPV1 ion channel antagonist capsazepine inhibits osteoclast and osteoblast differentiation in vitro and ovariectomy induced bone loss in vivo. Bone 46(4): 10891099.

47. AL M, HA L, Liedtke W (2015) TRPV4 as a therapeutic target for joint diseases. Naunyn Schmiedebergs Arch. Pharmacol 388(4): 437-450.

48. Christopher JO Conor, Griffin TM, Liedtke W (2013) Increased susceptibility of Trpv4-deficient mice to obesity and obesity-induced osteoarthritis with very high-fat diet. Ann Rheum Dis 72(2): 300-304.

49. Kajiya H, Okamoto F, Nemoto T (2010) RANKL-induced TRPV2 expression regulates osteoclastogenesis via calcium oscillations. Cell Calcium 48(5): 260-269.

$\begin{array}{ll}\text { BIOMEDICAL } & \text { Assets of Publishing with us } \\ \text { RESEARCHES } & \text { - Global archiving of articles } \\ & \text { - Immediate, unrestricted online access } \\ & \text { - Rigorous Peer Review Process } \\ \end{array}$

